\title{
Adjuvant endocrine therapy in premenopausal women with breast cancer
}

\author{
M. Gnant, P. Dubsky, F. Fitzal, P. Blaha, G. Steger, R. Jakesz \\ Department of Surgery, Medical University of Vienna, Vienna, Austria.
}

\begin{abstract}
Breast cancer is the most common malignancy among women worldwide, and a quarter of all breast cancers are diagnosed in premenopausal women. Adjuvant chemotherapy is well established as the therapy of choice for hormone receptor-negative breast cancers. Historically, ovarian ablation substantially inhibited the progression of hormone-responsive breast cancer. Current adjuvant treatment options for premenopausal patients with hormone receptor-positive breast cancer include endocrine therapy and chemotherapy. Pharmacologic ovarian suppression alone has limited efficacy in the adjuvant setting, but it offers good clinical outcomes when combined with tamoxifen or chemotherapy. In recent years, aromatase inhibitors (Als) have become the endocrine therapy of choice in postmenopausal women with hormoneresponsive breast cancer, but their efficacy in the premenopausal setting is yet to be established. However, in a large, phase III randomized study (Austrian Breast and Colorectal Cancer Study Group Trial 12) that matured earlier this year, the combination of ovarian suppression and Al, anastrozole, did not show superior efficacy compared with ovarian suppression plus tamoxifen, but was associated with fewer serious adverse effects in premenopausal women with early stage hormone-responsive breast cancer. Data from ongoing and future trials will help to further define the role of ovarian suppression and Als in the adjuvant setting for premenopausal breast cancer.
\end{abstract}

Keywords: Adjuvant therapy; Breast cancer; LHRH agonist; Ovarian suppression; Premenopausal

\section{Introduction}

Breast cancer is the most prevalent malignancy in women worldwide. The global incidence of breast cancer was estimated at more than 1.3 million new cases in 2007, and nearly 465000 women were expected to die because of this disease [1]. The incidence of breast cancer increases with age - in

Correspondence to: Michael Gnant, MD, Department of Surgery, Medical University of Vienna, Währinger Gürtel 18-20, A-1090, Vienna, Austria. E-mail: michael.gnant@meduniwien.ac.at; Tel: +43 140400 5646; Fax: $+431404006807$

Received: $14 / 10 / 08$

Accepted: 16/01/09

First published online 18/06/09

BCO/799/2008/FO the United States, the 10-year probability of developing invasive breast cancer increases from one in 234 for a 30 -year-old woman to one in 28 for a 60 -year-old woman [2]. In spite of the striking correlation between advanced age and risk of invasive breast cancer, approximately one quarter of all breast cancers are diagnosed in premenopausal women [3]. The last few decades have seen great improvements in the early detection of breast tumors, and as a result, the majority of breast cancers are diagnosed at early stages, allowing for earlier and more effective therapy [2]. However, clinical outcomes can be improved further. For instance, the 5-year survival rate is lower for women with breast cancer diagnosed before they are 40 years old $(80 \%)$ compared with those diagnosed at ages 40 and older (89\%), potentially because 
younger women present with more aggressive disease at the time of diagnosis [2].

The expression of estrogen and/or progesterone receptors in tumor cells is a major determinant of therapeutic options for breast cancer. Hormone receptor-negative breast cancers, which are more common in premenopausal compared with postmenopausal women, are typically treated using cytotoxic chemotherapy. However, approximately $60 \%$ of breast cancers detected in premenopausal women are hormone receptor-positive $\left(\mathrm{HR}^{+}\right)$, and will likely respond to endocrine therapy [3]. Although the efficacy of surgical ovarian ablation (oophorectomy) for targeted treatment of breast cancer has been known for more than a century, progress in improving endocrine therapies in the premenopausal setting has been slow [4].

\section{Ovarian ablation in premenopausal women with breast cancer}

Estrogen production in premenopausal women is predominantly ovarian, with a small component contributed by aromatization reactions in extragonadal tissues. In contrast, aromatase activity in peripheral tissues is the main source of estrogen in postmenopausal women [5]. As a result, aromatase inhibitors (Als) effectively suppress estrogen production in postmenopausal women, whereas suppression of estrogen production in premenopausal women requires ovarian ablation. Permanent ovarian ablation can be achieved by surgery (bilateral oophorectomy) or radiation therapy; cytotoxic agents such as cyclophosphamide can also induce ovarian dysfunction [5]. Bilateral oophorectomy is the preferred means of achieving permanent ovarian ablation because it leads to a rapid and irreversible decrease in circulating estrogen levels and also reduces the risk of ovarian cancer in women who are at elevated risk for that disease [5].

Although early trials of ovarian ablation did not reveal an immediate benefit in the premenopausal breast cancer setting, a meta-analysis of data from various trials in patients with early breast cancer (with or without chemotherapy; total $N>2200$ ) revealed that ovarian ablation resulted in significant improvements in recurrence-free survival (RFS) and overall survival (OS) compared with patients who did not undergo ovarian ablation [6]. Overall improvement in RFS in the ovarian ablation groups was $18.5 \% \quad(P=0.0007)$. The effect of ovarian ablation was much more dramatic in patients who did not receive chemotherapy: ovarian ablation improved RFS by $25 \%(P=0.0005)$ in this cohort vs. $10 \%$ (statistically not significant) in the chemotherapy cohort [6]. Overall survival was similarly improved by ovarian ablation. The benefits of ovarian ablation were more substantial in patients with node-positive disease compared with nodenegative: relative improvements in disease-free survival (DFS) and OS were $13.4 \%$ and $12.5 \%$, respectively, for node-positive cancers and $8.9 \%$ and $5.6 \%$, respectively, for node-negative cancers $(P \leqslant 0.01$ vs. no ablation for all) [6]. Conversely, improvements in RFS and OS because of ovarian ablation were not significant in 1354 women aged 50 years or older (perimenopausal population) [7]. In another trial in 762 premenopausal women with node-positive, $\mathrm{HR}^{+}$breast cancer, ovarian ablation and chemotherapy provided similar DFS and OS benefits [8]. However, chemotherapy was associated with more adverse events: $57 \%$ of patients in the chemotherapy arm experienced at least one episode of myelosuppression, and $33 \%$ of patients had moderate to severe nausea and vomiting in spite of prophylactic antiemetics [8]. Thus, in this study, ovarian ablation provided survival benefits similar to those of cytotoxic chemotherapy with less toxicity.

It is also important to note that adjuvant chemotherapy frequently leads to ovarian shutdown in premenopausal women. The rate of chemotherapyinduced ovarian shutdown is influenced by the chemotherapy regimen used, the number of cycles, and the patient's condition and age [9]. For example, 96\% of women between the ages of 40 and 49 years experienced ovarian failure after doxorubicin-based chemotherapy compared with only $33 \%$ of women between the ages of 30 and 39 years [9]. Similarly, among women receiving epirubicin-based chemotherapy, rates of amenorrhea were $88 \%$ in women 40 years of age or older vs. $32 \%$ in women between the ages of 32 and 39 years [9]. Therefore, it is likely that improved clinical outcomes after adjuvant chemotherapy are at least partly attributable to the indirect endocrine effects of these treatments, especially in older pre- and perimenopausal women. Furthermore, a retrospective analysis of 3700 premenopausal women who underwent adjuvant chemotherapy for breast cancer showed that clinical outcomes were significantly worse in younger women ( $<35$ years old): 10 -year DFS was $35 \%$ for women under the age of 35 years vs. $47 \%$ for older women $(P<0.001)[10]$. Interestingly, DFS was substantially lower in young premenopausal women receiving chemotherapy for $\mathrm{HR}^{+}$(hormone-responsive) tumors compared with hormone receptor-negative tumors [10]. Taken together with the lower rates of chemotherapy-induced ovarian shutdown in younger women, these data indicate that young premenopausal women with $\mathrm{HR}^{+}$breast cancer may benefit from endocrine therapy. 


\section{Reversible ovarian suppression as adjuvant therapy for premenopausal women with breast cancer}

Reversible ovarian suppression (sometimes also called medical castration) can be achieved through treatment with luteinizing hormone-releasing hormone $(\mathrm{LHRH})$ analogues. These agents act via pituitary LHRH receptors to suppress gonadotropin secretion, leading to dramatic reductions in the production of steroid hormones by the ovary [5]. This modality of ovarian suppression is generally reversible, thereby preserving fertility in young women undergoing adjuvant therapy for breast cancer. The efficacy of goserelin was similar to that of ovarian ablation (oophorectomy or radiation) in a small prospective trial in 85 perimenopausal women with metastatic breast cancer [11]. As a result, more recent studies have included treatment with goserelin or other LHRH inhibitors (e.g., leuprolide and triptorelin) as a means of suppressing ovarian function in pre- and perimenopausal women with breast cancer.

\section{Trials comparing reversible ovarian sup- pression to chemotherapy in the adjuvant setting for premenopausal breast cancer}

\section{The Zoladex Early Breast Cancer Research Association (ZEBRA) trial}

The ZEBRA study evaluated the efficacy of ovarian suppression with goserelin $(3.6 \mathrm{mg}$ subcutaneous (SC) every 28 days; $n=817$ ) compared with six cycles of CMF (cyclophosphamide, methotrexate, and fluorouracil) chemotherapy $(n=823)$ as adjuvant therapy for node-positive breast cancer in premenopausal women [12]. After a median follow-up at 6 years, DFS and OS were similar in both treatment arms for patients with estrogen receptor-positive $\left(\mathrm{ER}^{+}\right)$tumors (goserelin vs. CMF: hazard ratio $(\mathrm{HR})=1.01$ for DFS and 0.99 for OS). However, goserelin was significantly inferior to CMF in the estrogen receptor-negative cohort $(\mathrm{HR}=1.76, P=0.0006$ for $\mathrm{DFS}$ and $\mathrm{HR}=1.77$, $P=0.0043$ for OS) [12]. Amenorrhea was prevalent in the goserelin arm during treatment but was reversed in more than $75 \%$ of patients within 1 year after stopping goserelin. Although fewer patients in the CMF arm developed amenorrhea during treatment, the amenorrhea in this group persisted for years after completion of therapy [12].

\section{The Takeda Adjuvant Breast Cancer Study with Leuprorelin Acetate (TABLE) trial}

This study evaluated the efficacy of ovarian suppression using the LHRH agonist leuprorelin acetate (SC every 3 months for 2 years; $n=299$ ) with six cycles of standard CMF chemotherapy $(n=300)$ in premenopausal women with node-positive breast cancer $[13,14]$. At a median follow-up of 5.8 years, RFS was similar in the two treatment arms $(\mathrm{HR}=$ 1.19; $P=0.15$ ), whereas exploratory analysis of OS favors the endocrine therapy arm $(H R=1.50$; $P=0.005$ ) [14]. These data show that ovarian suppression using LHRH agonists has efficacy comparable with that of chemotherapy in premenopausal women with endocrine-responsive breast cancer.

\section{Tamoxifen as adjuvant therapy for pre- menopausal women with breast cancer}

Tamoxifen is a selective estrogen receptor modulator that has been used extensively to treat both metastatic and early stage hormone-responsive breast cancer. The Early Breast Cancer Trialists' Collaborative Group (EBCTCG) compounded a recent overview of several trials of tamoxifen in the adjuvant setting. The 'control' arms in these studies varied from no adjuvant therapy in some to active chemotherapy in others, but all were characterized by the absence of adjuvant tamoxifen. Outcomes of these meta-analyses revealed that 5 years of adjuvant tamoxifen treatment reduces the annual death rate attributable to breast cancer by approximately $31 \%$ vs. control in patients with $\mathrm{ER}^{+}$disease, and this effect is largely independent of age, adjuvant chemotherapy, progesterone-receptor status, or other tumor characteristics [15]. In pooled data from 44 trials (total $N>33000$ ), tamoxifen treatment for 1 to 2 years significantly decreased breast cancer recurrence and mortality in women with $\mathrm{ER}^{+}$disease (tamoxifen vs. control: $\mathrm{HR}=0.74$ for recurrence and 0.82 for OS; $P<0.00001$ for both) [15]. Data from 12 trials of tamoxifen treatment for 5 years (total $N>15000$ ) yielded even greater improvements in clinical outcomes (tamoxifen vs. control: RFS HR= 0.59 and $\mathrm{OS} \mathrm{HR}=0.66 ; P<0.00001$ for both) [15]. Tamoxifen treatment for this duration also significantly decreased the annual incidence of contralateral breast carcinomas from $0.6 \%$ (in control arm) to $0.4 \%(P<0.00001)$. Approximately 5 years of tamoxifen treatment improved RFS and OS in women with $\mathrm{ER}^{+}$disease irrespective of age: HRs for RFS were 0.56 for age less than 40 years, 0.71 for ages 41 to 49 years, 0.66 for ages 50 to 59 years, and 0.55 for ages 60 to 69 years $(P<0.00001$ for tamoxifen vs. control in all strata) [15].

It is therefore evident from the data described that tamoxifen substantially improves clinical outcomes and survival for premenopausal women with hormone-responsive breast cancer compared with controls. However, tamoxifen treatment is associated with a significant increase in uterine cancers: in the 
Table 1. Clinical trials evaluating the efficacy of ovarian suppression as combination or sequential therapy for hormone receptorpositive breast cancer in premenopausal women.

\begin{tabular}{|c|c|c|c|}
\hline Trial & Treatments compared & $N$ & Outcome \\
\hline IBCSG-VIII [17] & $\begin{array}{l}\text { CMF }(\times 6) \text { vs. Gos }(24 \text { months }) \text { vs. CMF }(\times 6) \\
\text { then Gos (18 months) }\end{array}$ & 1063 & $\begin{array}{l}\text { DFS in patients with } \mathrm{ER}^{-} \text {tumors: } \\
\text { CMF }>\text { Gos } \\
\text { DFS in patients with } \mathrm{ER}^{+} \text {tumors: } \\
\text { CMF }=\text { Gos } \\
\text { CMF then Gos }>\text { CMF } \\
\text { CMF then Gos }>\text { Gos }\end{array}$ \\
\hline INT $0101[18]$ & $\begin{array}{l}\text { CAF }(\times 6) \text { vs. CAF }(\times 6) \text { then Gos }(60 \text { months) vs. } \\
\text { CAF }(\times 6) \text { then Gos }+ \text { Tam (both } 60 \text { months) }\end{array}$ & 1503 & $\begin{array}{l}\text { DFS, OS, and TTR: } \\
\text { CAF then Gos + Tam }>\text { CAF then Gos }>\text { CAF }\end{array}$ \\
\hline IBCAG-02 [19] & CMF $(\times 6)$ vs. OFS $^{a}+$ Tam $(60$ months $)$ & 244 & $\begin{array}{l}\text { Similar DFS and OS } \\
\quad \text { Amenorrhea } \uparrow \text { OS in CMF arm }\end{array}$ \\
\hline ABCSG-5 [20] & $\begin{array}{l}\text { CMF }(\times 6) \text { vs. Endocrine (Gos }+ \text { Tam (36 months) } \\
\text { then Tam alone }(24 \text { months)) }\end{array}$ & 1034 & $\begin{array}{l}\text { RFS, local RFS, and OS: } \\
\text { Endocrine }>\text { CMF }\end{array}$ \\
\hline ABCSG-12 [27] & Gos + Tam vs. Gos + Al; all treatments for 36 months & 1803 & Similar DFS \\
\hline \multicolumn{4}{|l|}{ Ongoing } \\
\hline SOFT & OFS $^{a}+$ Al vs. OFS ${ }^{a}+$ Tam vs. Tam; all for 60 months & 3000 & \\
\hline TEXT & $\mathrm{OFS}^{\mathrm{a}}+\mathrm{Al}$ vs. $\mathrm{OFS}^{\mathrm{a}}+$ Tam; both for 60 months & 2600 & \\
\hline
\end{tabular}

IBCSG: International Breast Cancer Study Group; CMF: cyclophosphamide, methotrexate, and fluorouracil chemotherapy; $\times 6$ : 6 cycles of chemotherapy; Gos: goserelin; DFS: disease-free survival; ER: estrogen receptor; INT: Intergroup Trial; CAF: cyclophosphamide, doxorubicin, and fluorouracil chemotherapy; Tam: tamoxifen; OS: overall survival; TTR: time to recurrence; IBCAG: Italian Breast Cancer Adjuvant Group; OFS: ovarian function suppression; ABCSG: Austrian Breast and Colorectal Cancer Study Group; RFS: recurrence-free survival; Al: aromatase inhibitor; SOFT: Suppression of Ovarian Function Trial; TEXT: Tamoxifen and Exemestane Trial.

${ }^{a}$ Ovarian-function suppression with bilateral oophorectomy, radiation to the ovaries, or monthly administration of luteinizing hormone-releasing hormone agonists for 5 years.

pooled analysis described above, the annual rate of uterine cancer in patients receiving 5 years of tamoxifen $(n=7512)$ was $0.19 \%$ compared with $0.06 \%$ in controls $(n=7505 ; P<0.00001)$ [15]. In the same analysis, there was also a trend toward an increased incidence of stroke in patients receiving tamoxifen for 5 years (54 vs. 29 controls; $P=0.07$ ) [15]. Therefore, other options for adjuvant endocrine therapy should be investigated in the premenopausal breast cancer setting.

\section{Sequential and combination adjuvant endocrine therapies in the premenopausal breast cancer setting}

Ovarian suppression and adjuvant chemotherapy individually decrease disease recurrence and improve survival in premenopausal women with hormoneresponsive breast cancer. Further, pooled data from several randomized clinical trials projected that sequential therapy with six cycles of anthracyclinebased adjuvant chemotherapy followed by 5 years of tamoxifen will reduce 15-year mortality because of $\mathrm{HR}^{+}$breast cancer by approximately 50\% [15]. In addition, a meta-analysis of four trials (combined $N=506$ ) of tamoxifen plus LHRH agonists vs. LHRH agonists alone in the advanced breast cancer setting in premenopausal women revealed that the combined endocrine therapy significantly improved OS $(\mathrm{HR}=$ $0.78 ; P=0.02)$ and progression-free survival $(\mathrm{HR}=$
$0.70 ; P=0.0003)$ [16]. However, the effects of adjuvant therapies plus ovarian suppression (concomitant or sequential) in the early breast cancer setting were less clear. The following section will summarize key findings from trials (Table 1) that evaluated sequential and combination chemoendocrine therapy regimens.

\section{Trials evaluating sequential chemo- and endocrine therapies}

\section{International Breast Cancer Study Group (IBCSG) trial VIII}

The IBCSG trial VIII investigated the efficacy of six cycles of CMF chemotherapy $(n=360)$ vs. ovarian suppression with goserelin for 2 years $(n=346)$ vs. CMF (six cycles) followed by goserelin (for 18 months; $n=357$ ) in pre- and perimenopausal women with node-negative breast cancer [17]. At a median follow-up of 7 years, DFS in the hormone receptornegative subgroup was higher with CMF (alone or followed by goserelin) compared with goserelin alone. In contrast, in the $\mathrm{HR}^{+}$cohort, CMF and goserelin each provided similar DFS benefits (5-year DFS $=81 \%$ for both groups) and the sequential therapy improved DFS (5-year DFS $=86 \%$ ) compared with either modality alone [17]. The improvement in DFS with sequential therapy was especially marked in younger women ( $\leqslant 39$ years old) with $\mathrm{HR}^{+}$ tumors. In this group, the DFS HR for sequential therapy vs. monotherapy was $0.34(P=0.02)$ [17]. 
Eastern Cooperative Oncology Group/ US Intergroup Trial INT 0101

This study compared the efficacy of chemotherapy (six cycles of cyclophosphamide, doxorubicin, and fluorouracil (CAF)) alone $(n=494)$, CAF followed by 5 years of monthly goserelin $(n=502)$, or CAF followed by 5 years of monthly goserelin and daily tamoxifen $(n=507)$ in premenopausal women with $\mathrm{HR}^{+}$breast cancer [18]. At a median follow-up of 9.6 years, sequential therapy with CAF and goserelin produced non-significant improvements in DFS, OS, and time to recurrence compared with CAF alone. In contrast, sequential therapy with $\mathrm{CAF}$, goserelin, and tamoxifen significantly improved DFS $(\mathrm{HR}=0.74$; $P<0.01)$ and time to recurrence $(\mathrm{HR}=0.73 ; P<0.01)$ compared with CAF followed by goserelin alone [18].

\section{Trials evaluating combination endocrine therapies vs. chemotherapy}

\section{Italian Breast Cancer Adjuvant Group 02 (IBCAG-02) randomized trial}

The IBCAG-02 trial compared the efficacy of six cycles of standard CMF chemotherapy $(n=120)$ with 5 years of ovarian suppression plus daily tamoxifen $(n=124)$ [19]. Ovarian suppression was achieved by oophorectomy $(n=6)$, irradiation $(n=31)$, or goserelin injections $(n=87)$. At a median follow-up of 76 months, DFS and OS were comparable between groups. No significant differences in DFS or OS were observed between treatments after controlling for age, tumor size, and nodal status. However, treatment-induced amenorrhea was associated with significantly better OS within the CMF arm $(P=0.05)$ [19].

\section{Austrian Breast and Colorectal Cancer Study Group Trial 5 (ABCSG-5)}

The ABCSG-5 trial randomized 1034 premenopausal women with hormone-responsive breast cancer to either six cycles of CMF chemotherapy $(n=523)$ or endocrine therapy (3 years of goserelin plus tamoxifen followed by 2 years of tamoxifen alone; $n=511$ ) [20]. At a median follow-up of 60 months, RFS and local recurrence-free survival differed significantly in favor of endocrine therapy $(\mathrm{HR}=1.40, P=0.019$ for RFS; $\mathrm{HR}=1.98, P=0.008$ for local recurrence-free survival; both HRs are for chemotherapy vs. endocrine therapy). Overall survival was also improved in the endocrine-therapy arm compared with CMF, but the difference was not statistically significant [20].

A recent meta-analysis of data from 16 trials involving 11906 premenopausal women with early breast cancer confirmed that LHRH agonists (such as goserelin) have similar efficacy to chemotherapy in $\mathrm{ER}^{+}$tumors but are ineffective against estrogen receptor-negative tumors [21]. The addition of LHRH agonists to tamoxifen, chemotherapy, or both significantly reduced disease recurrence and death after recurrence vs. each regimen without LHRH agonists (12.7\% decrease, $P=0.02$ and $15.1 \%$ decrease, $P=0.03$, respectively) [21]. Thus, LHRH agonists represent an important addition to adjuvant therapy for hormone-responsive breast cancer in the premenopausal setting.

\section{Aromatase inhibitors in the premenopausal breast cancer setting}

Aromatase inhibitors have now become the standard option for adjuvant endocrine therapy in postmenopausal women with $\mathrm{HR}^{+}$breast cancer, although questions regarding the optimal timing and duration of Al therapy in this setting remain [22]. In the Breast International Group (BIG) 1-98 study (a randomized, double-blind, phase III trial in 8010 postmenopausal women), the highly active thirdgeneration Al, letrozole, significantly improved DFS $(\mathrm{HR}=0.81, P=0.003$ at a follow-up of 26 months; $\mathrm{HR}=0.82, P=0.007$ at a follow-up of 51 months) and reduced the incidence of early distant metastases (HR $=0.73, P=0.001$ at 26 months follow-up) $[23,24]$. The use of Als in the premenopausal setting has yet to be established. A major concern about Al therapy in premenopausal women is their potential for hyperstimulation of ovarian function - reduction in estrogen levels because of $\mathrm{Al}$ therapy can lead to a reflex increase in gonadotropin production, resulting in ovarian stimulation [22]. However, the efficacy of Als combined with chronic ovarian suppression is being evaluated. Recently, the HOBOE (Hormonal Adjuvant Treatment Bone Effects) study completed analysis of the endocrine effects of letrozole in combination with ovarian suppression (using triptorelin administered every 4 weeks) vs. tamoxifen plus triptorelin in the premenopausal cohort $(N=81$ women with operable, $\mathrm{HR}^{+}$breast cancer) [25]. After 6 months of adjuvant endocrine therapy, median serum estradiol levels were significantly lower in the letrozole-plus-triptorelin arm vs. tamoxifen plus triptorelin ( $<5$ vs. $7.95 \mathrm{pg} / \mathrm{ml} ; P=0.0008)$ [25]. Clinical outcomes and skeletal health data from this study will be forthcoming. The combination of goserelin and the Al, anastrozole, brought about a similar substantial reduction in circulating estradiol levels in a small pilot study $(n=16)$ [26]. Recently, the ABCSG-12 trial evaluated the safety and efficacy of the goserelin-anastrozole combination in $>1800$ premenopausal women with early stage, hormoneresponsive breast cancer [27]. 


\section{Austrian Breast and Colorectal Cancer Study Group Trial 12 (ABCSG-12)}

The ABCSG-12 study randomized 1803 premenopausal women with early stage (I/II) breast cancer to 3 years of treatment with monthly goserelin plus daily tamoxifen or anastrozole, alone or in combination with twice-yearly doses of zoledronic acid (a bisphosphonate) [27]. The primary endpoint was two pairwise comparisons of DFS: the first was the goserelin-plus-anastrozole group vs. goserelin-plus-tamoxifen group, whereas the second was the endocrine therapy-plus-zoledronicacid group vs. the endocrine therapy-alone group. Secondary endpoints included RFS and OS. Although event-driven analysis at a median followup of 47.8 months revealed that anastrozole was not superior to tamoxifen, there was no significant difference in efficacy between the anastrozole and tamoxifen arms in this patient population $(\mathrm{HR}=$ $1.10, P=0.59$ for DFS) [27]. However, in keeping with the known safety profiles of these agents, patients receiving anastrozole experienced fewer endometrial abnormalities (e.g., uterine polyps) and thromboembolic adverse events compared with tamoxifen. Longer follow-up of this trial is needed to elucidate possible differences between anastrozole and tamoxifen in long-term efficacy and safety outcomes. Interestingly, the addition of twice-yearly zoledronic acid to adjuvant endocrine therapy not only prevented bone loss [28] but also significantly improved DFS $(\mathrm{HR}=0.64 ; P=0.012)$ and RFS $(\mathrm{HR}=0.65 ; P=0.014)$ compared with endocrine therapy alone [27]. These results indicate that concomitant treatment with bisphosphonates such as zoledronic acid to preserve bone health during adjuvant endocrine therapy for premenopausal breast cancer may provide additional benefits including substantially improved clinical outcomes, potentially because of the inherent antitumor properties of zoledronic acid and other bisphosphonates [29].

The efficacy of ovarian suppression combined with Als in the premenopausal setting is currently being further evaluated. Ongoing phase II studies in the premenopausal metastatic (stage IV) breast cancer setting include trials NCT00498901 in the United States (sample size, 25) comparing letrozole plus goserelin with letrozole plus leuprolide [30] and NCT00532272 in Korea (sample size, 70) comparing letrozole plus goserelin in premenopausal women with letrozole alone in postmenopausal women [31]. In the adjuvant setting for early stage breast cancer, ongoing phase III trials evaluating ovarian suppression plus Als involve $>5000$ premenopausal women. The SOFT (Suppression of Ovarian Function) trial is ongoing and will enroll
3000 patients [32]. This study will compare the efficacy of ovarian suppression plus the Al exemestane vs. ovarian suppression plus tamoxifen vs. tamoxifen alone. Another ongoing trial with similar design, TEXT (Tamoxifen and Exemestane Trial), will compare ovarian suppression plus exemestane with ovarian suppression plus tamoxifen in more than 2600 premenopausal women with early stage breast cancer [33]. Data from these trials will further elucidate the therapeutic potential of Als in the adjuvant as well as advanced settings for premenopausal breast cancer.

\section{Conclusions}

A large body of data shows that adjuvant endocrine therapy for premenopausal women with hormoneresponsive breast cancer can achieve clinical outcomes similar to that of cytotoxic chemotherapy while sparing these patients the toxicities and adverse events associated with chemotherapy. Moreover, in recent years, it has also become evident that the combination of standard adjuvant therapy with chronic, reversible ovarian suppression (e.g., using LHRH agonists) can further improve DFS and OS in this setting. Although the adjuvant role of Als is yet to be established in premenopausal women undergoing chronic ovarian suppression, it is possible that potent, third-generation Als may provide benefits beyond those obtained with tamoxifen in this setting. Future data from ongoing trials will help to further understand the utility of Als in the adjuvant setting for premenopausal breast cancer. Interestingly, recent data reveal that the addition of bisphosphonates to adjuvant endocrine therapy not only prevents Al-associated bone loss but also substantially improves clinical outcomes for premenopausal women with early stage breast cancer. Therefore, such combination therapies might, in future, provide the best overall outcomes in this setting.

\section{Acknowledgements}

The ABCSG-12 clinical trial was supported by AstraZeneca and Novartis Pharmaceuticals. Financial support for medical editorial assistance was provided by Novartis Pharmaceuticals. We thank Shalini Murthy, PhD, for her medical editorial assistance with this manuscript.

Conflict of interest: Dr Gnant has served on advisory boards for and received consulting fees from AstraZeneca, Novartis, and Pfizer and lecture fees from Roche, Schering, Pfizer, Novartis, AstraZeneca, sanofi-aventis, and Amgen. 


\section{References}

1. Garcia M, Jemal A, Ward EM, et al. Global Cancer Facts \& Figures 2007. Atlanta, GA: American Cancer Society, 2007.

2. American Cancer Society. Breast Cancer Facts \& Figures 2007-2008. Atlanta: American Cancer Society, Inc., 2007.

3. Anderson WF, Chatterjee N, Ershler WB, Brawley OW. Estrogen receptor breast cancer phenotypes in the Surveillance, Epidemiology, and End Results database. Breast Cancer Res Treat 2002; 76: 27-36.

4. Pritchard K. Endocrinology and hormone therapy in breast cancer: endocrine therapy in premenopausal women. Breast Cancer Res 2005; 7: 70-76.

5. Emens LA, Davidson NE. Adjuvant hormonal therapy for premenopausal women with breast cancer. Clin Cancer Res 2003; 9: 486S-494S.

6. Early Breast Cancer Trialists' Collaborative Group. Ovarian ablation in early breast cancer: overview of the randomised trials. Lancet 1996; 348: 1189-1196.

7. Early Breast Cancer Trialists' Collaborative Group. Ovarian ablation for early breast cancer. Cochrane Database Syst Rev 2000: CD000485.

8. Ejlertsen B, Mouridsen HT, Jensen MB, et al. Similar efficacy for ovarian ablation compared with cyclophosphamide, methotrexate, and fluorouracil: from a randomized comparison of premenopausal patients with node-positive, hormone receptor-positive breast cancer. $J$ Clin Oncol 2006; 24: 4956-4962.

9. Walshe JM, Denduluri N, Swain SM. Amenorrhea in premenopausal women after adjuvant chemotherapy for breast cancer. J Clin Oncol 2006; 24: 5769-5779.

10. Aebi S, Gelber S, Castiglione-Gertsch $\mathrm{M}$, et al. Is chemotherapy alone adequate for young women with oestrogen-receptor-positive breast cancer? Lancet 2000; 355: 1869-1874.

11. Boccardo F, Rubagotti A, Perrotta A, et al. Ovarian ablation versus goserelin with or without tamoxifen in pre-perimenopausal patients with advanced breast cancer: results of a multicentric Italian study. Ann Oncol 1994; 5: 337-342.

12. Jonat W, Kaufmann M, Sauerbrei W, et al. Goserelin versus cyclophosphamide, methotrexate, and fluorouracil as adjuvant therapy in premenopausal patients with nodepositive breast cancer: The Zoladex Early Breast Cancer Research Association Study. J Clin Oncol 2002; 20: 4628-4635.

13. Schmid P, Untch M, Wallwiener D, et al. Cyclophosphamide, methotrexate and fluorouracil (CMF) versus hormonal ablation with leuprorelin acetate as adjuvant treatment of node-positive, premenopausal breast cancer patients: preliminary results of the TABLE-study (Takeda Adjuvant Breast cancer study with Leuprorelin Acetate). Anticancer Res 2002; 22: 2325-2332.

14. Schmid P, Untch M, Kosse V, et al. Leuprorelin acetate every-3-months depot versus cyclophosphamide, methotrexate, and fluorouracil as adjuvant treatment in premenopausal patients with node-positive breast cancer: the TABLE study. J Clin Oncol 2007; 25: 2509-2515.
15. Early Breast Cancer Trialists' Collaborative Group (EBCTCG). Effects of chemotherapy and hormonal therapy for early breast cancer on recurrence and 15year survival: an overview of the randomised trials. Lancet 2005; 365: 1687-1717.

16. Klijn JGM, Blamey RW, Boccardo F, Tominaga T, Duchateau L, Sylvester R. Combined tamoxifen and luteinizing hormone-releasing hormone (LHRH) agonist versus LHRH agonist alone in premenopausal advanced breast cancer: a meta-analysis of four randomized trials. J Clin Oncol 2001; 19: 343-353.

17. Castiglione-Gertsch $M$, O'Neill $A$, Price $\mathrm{KN}$, et al. Adjuvant chemotherapy followed by goserelin versus either modality alone for premenopausal lymph nodenegative breast cancer: a randomized trial. J Natl Cancer Inst 2003; 95: 1833-1846.

18. Davidson NE, O'Neill AM, Vukov AM, et al. Chemoendocrine therapy for premenopausal women with axillary lymph node-positive, steroid hormone receptor-positive breast cancer: results from INT 0101 (E5188). J Clin Oncol 2005; 23: 5973-5982.

19. Boccardo F, Rubagotti A, Amoroso D, et al. Cyclophosphamide, methotrexate, and fluorouracil versus tamoxifen plus ovarian suppression as adjuvant treatment of estrogen receptor-positive pre-/perimenopausal breast cancer patients: results of the Italian Breast Cancer Adjuvant Study Group 02 randomized trial. J Clin Oncol 2000; 18: 2718-2727.

20. Jakesz R, Hausmaninger $\mathrm{H}$, Kubista $\mathrm{E}$, et al. Randomized adjuvant trial of tamoxifen and goserelin versus cyclophosphamide, methotrexate, and fluorouracil: evidence for the superiority of treatment with endocrine blockade in premenopausal patients with hormone-responsive breast cancer - Austrian Breast and Colorectal Cancer Study Group Trial 5. J Clin Oncol 2002; 20: 4621-4627.

21. Cuzick J, Ambroisine L, Davidson N, et al. Use of luteinising-hormone-releasing hormone agonists as adjuvant treatment in premenopausal patients with hormone-receptor-positive breast cancer: a meta-analysis of individual patient data from randomised adjuvant trials. Lancet 2007; 369: 1711-1723.

22. Winer EP, Hudis C, Burstein HJ, et al. American Society of Clinical Oncology technology assessment on the use of aromatase inhibitors as adjuvant therapy for postmenopausal women with hormone receptor-positive breast cancer: status report 2004. J Clin Oncol 2005; 23: 619-629.

23. Coates AS, Keshaviah A, Thurlimann B, et al. Five years of letrozole compared with tamoxifen as initial adjuvant therapy for postmenopausal women with endocrineresponsive early breast cancer: update of study BIG 1-98. J Clin Oncol 2007; 25: 486-492.

24. Thurlimann B, Keshaviah A, Coates AS, et al. A comparison of letrozole and tamoxifen in postmenopausal women with early breast cancer. $N$ Engl $J$ Med 2005; 353: 2747-2757.

25. Rossi E, Morabito A, De Maio E, et al. Endocrine effects of adjuvant letrozole + triptorelin compared with tamoxifen + triptorelin in premenopausal patients with early breast cancer. J Clin Oncol 2008; 26: 264-270.

26. Forward DP, Cheung KL, Jackson L, Robertson JF. Clinical and endocrine data for goserelin plus anastrozole as 
second-line endocrine therapy for premenopausal advanced breast cancer. Br J Cancer 2004; 90: 590-594.

27. Gnant MF, Mlineritsch B, Schippinger W, et al. Adjuvant ovarian suppression combined with tamoxifen or anastrozole, alone or in combination with zoledronic acid, in premenopausal women with endocrine-responsive, stage I and II breast cancer: first efficacy results from ABCSG-12 (ASCO abstract LBA4). J Clin Oncol 2008; 26(Suppl): 6s.

28. Gnant M, Mlineritsch B, Luschin-Ebengreuth $G$, et al. Adjuvant endocrine therapy plus zoledronic acid in premenopausal women with early-stage breast cancer: 5-year follow-up of the ABCSG-12 bone-mineral density substudy. Lancet Oncol 2008; 9: 840-849.

29. Winter MC, Holen I, Coleman RE. Exploring the antitumour activity of bisphosphonates in early breast cancer. Cancer Treat Rev 2008; 34: 453-475.

30. US National Institutes of Health. Letrozole and goserelin or leuprolide in treating premenopausal estrogen receptor- positive patients with stage IV breast cancer. http:// clinicaltrials.gov/ct2/show/NCT00498901; updated 18 April 2009 (accessed 5 June 2009).

31. US National Institutes of Health. Goserelin plus letrozole in premenopausal patients versus letrozole alone in postmenopausal patients with metastatic breast cancer. http://clinicaltrials.gov/ct2/show/NCT00532272; published 19 September 2007 (accessed 5 June 2009).

32. US National Institutes of Health. Suppression of ovarian function plus either tamoxifen or exemestane compared with tamoxifen alone in treating premenopausal women with hormone-responsive breast cancer. http://clinicaltrials.gov/ct2/show/NCT00066690; updated 4 June 2009 (accessed 5 June 2009).

33. US National Institutes of Health. Triptorelin with either exemestane or tamoxifen in treating premenopausal women with hormone-responsive breast cancer. http:// clinicaltrials.gov/ct2/show/NCT00066703; updated 3 June 2009 (accessed 5 June 2009). 